\title{
Effect of Raman pump direction on conventional multiwavelength Brillouin-Raman fiber laser
}

\begin{abstract}
We have experimentally investigated the effects of Raman pump direction on the performance of multiwavelength Brillouin-Raman fiber laser. Comparison of Raman gain profiles at different Raman pump direction demonstrates higher gain in backward-pumping scheme than that in the forward pumping configuration. 192 channels at the typical Brillouin shifted frequency spacing of $10 \mathrm{GHz}$ are attained through backward pumping due to the higher Raman gain. On the other hand, forward-pumping scheme produces better optical signal-to-noise ratio of $26 \mathrm{~dB}$ with $20 \mathrm{GHz}$ channel spacing.
\end{abstract}

Keyword: Raman pump direction; Multiwavelength Brillouin-Raman fiber laser; Backward pumping 